\section{NEUROFEEDBACK AS A TREATMENT OF TICS IN CHILDREN}

doi:10.1136/archdischild-2012-302724.1494

'S Bakhtadze, 'N Geladze, 'N Khachapuridze, ${ }^{2} \mathrm{M}$ Nasrashvili. 'Tbilisi State Medical University; ${ }^{2}$ Cito', Medical Centre, Tbilisi, Georgia

Object: Tics are involuntary, sudden, rapid, recurrent, stereotyped motor movements or phonic productions that involve discrete muscle groups. Pharmacological treatment was considered as the most effective approach for tics management for many years. In recent period clinicians attempt to use behavioral methods for this purpose. The aim of our study was to use non pharmacological treatment like EEG biofeedback-neurofeedback (NF) for the treatment of tics.

Methods We have examined previously non treated 15 children ( 9 boys and 6 girls) with simple tics (average age 10 years). All children with complex tics and with other comorbidities were excluded from the study. Tics frequency and severity were assessed by Yale Global Tic Severity Scale (YGTSS). Sensorimotor rhythm (SMR) training was used for NF therapy. 30 session of NF with duration of 30 minutes of each was conducted in every patient. Data were analyzed by SPSS 10.0. ANOVA was used to determine the effect of treatment on YGTSS parameters.

Results The ANOVA showed a significant effect of treatment on YGTSS measures $(\mathrm{F}(1.37)=223.69$, MSE $=114.735, \mathrm{p}<.0001)$. These evidences suggest that NF significantly improves the severity and frequency of tics.

Conclusions Thus effectiveness of SMR training in children with tics is important as the drugs used for the treatment have severe side effects, compliance problems and etc. Cognitive behavioral therapy is effective not only for reducing of tics but also for increasing self esteem and social competence as well.

\section{ASSESSMENT OF ANTIPYRETICS AS SEIZURE PREVENTING IN FEBRILE CONVULSION}

doi:10.1136/archdischild-2012-302724.1495

A Mortazavi. Pediatrics, Social Security Organization/Imam Hossein Hospital, Zanjan, Iran

Background Febrile seizure is the most common seizure disorder during childhood. Antipyretics has not been shown to prevent seizure recurrences. (1)

Objectives Some researchers previously studied prophylactic efficacy of antipyretics in FC (2-7). Uhari studied synergic effect of antipyretics and BDPs in 1993. our study planned for antipyretics efficacy in FC in IRAN.

Methods Our observational, analytical, cross-sectional study was accomplished in over one year from 2009/Nov/23 to 2010/Nov/23. Sample size was 92 patients and sampling method was accidentally. Data collected by interview and analyzed using SPSS statistical software and Kolmogorov-smirnov, Pearson correlation and Regression tests.

Results 67 patients (72.8\%) had been received antipyretics before seizure occurrences, and 25 patients (27.2\%) hadn't. Antipyretic which had been used composed of one forms of acetaminophen in $62.7 \%$, NSAIDs in $4.5 \%$, and more than one drugs (mixed) in $32.8 \% .34$ patients $(50.7 \%)$ used antipyretics less than 3 hours, 31 patients $(46.3 \%)$ in $4-24 \mathrm{~h}$ and 2 patients (3\%) more than $24 \mathrm{~h}$ before seizure occurrences (FIG-1). (Table-1) shows maximal plasma concentration and plasma half-life of antipyretics. Approximately $50 \%$ of patients received antipyretics in appropriate time, but $25 \%$ treated after plasma half-life and remainder didn't received antipyretics before seizure occurrences.

Conclusion Preventable effects of antipyretics in FC, is in doubt but the difference between seizure occurrences in treated groups and remainders are not significant.

FIG-1: Antipyretic usage time before seizure occurrences.

\section{6 \\ WHEN SUSPECT PANAYIOTOPOULOS SYNDROME (PS) IN A CHILD WITH CLINICAL DIAGNOSIS OF GASTRIC ESOPHAGUS REFLUX DISEASE (GERD)}

doi:10.1136/archdischild-2012-302724.1496

C Pacchiarotti, P Parisi, A Ferretti, F Marra, S Bianchi, MP Villa. Sapienza University of Roma, Roma, Italy

Panayiotopoulos syndrome is "a benign age related focal seizure disorder occurring in early and mid-childhood. It is characterized by seizures, often prolonged, with predominantly autonomic symptoms, and by an EEG that shows shifting and/or multiple foci, often with occipital predominance" (definition of International League Against Epilepsy).

In literature the clinical features of PS was frequently mistaken as non-epileptic conditions such as acute encephalitis, syncope, migraine, cyclic vomiting syndrome, motion sickness, sleep disorder, or gastroenteritis.

In our experience during the last 2 years we have observed 4 children misdiagnosed by clinical history for GERD. Until now PS is underestimated with the consequences of high morbidity and costly mismanagement.

These 4 children were referred to general pediatrician at the age of 2-3 years because they started suffering from gastrointestinal emetic symptoms: nausea, retching, and vomiting. The attacks occurred mainly during sleep without sensory-motor clinical signs of seizure. One child also had monthly episodes of severe headache with clinical characteristics of migraine without aura.

After a treatment with proton pump inhibitors (PPI) and antiacid without benefits, according to the literature, they were sent to neurologist for suspection of PS which was confirmed by EEGs occipital features.

Because of the importance of the symptomatology, the children have been treated with Lamotrigine with benefit.

Conclusions When a child presents symptoms suggestive of GERD but non responding to specific GERD therapy, there is indication to effect an EEG to investigate a PS.

\section{THE EFFECT OF SURGERY TIME ON PROGNOSIS IN NEWBORNS WITH MENINGOMYELOCELE}

doi:10.1136/archdischild-2012-302724.1497

${ }^{1} \mathrm{MY}$ Oncel, ${ }^{1} \mathrm{R}$ Ozdemir, ${ }^{2} \mathrm{G}$ Kahilogulları, ${ }^{1} \mathrm{~S}$ Yurttutan, ${ }^{3} \mathrm{O}$ Erdeve; ${ }^{1,4} \mathrm{U}$ Dilmen. ${ }^{1}$ Neonatology, Zekai Tahir Burak Maternity Teaching Hospital; ${ }^{2}$ Neuorsurgery; ${ }^{3}$ Neonatology, Ankara University, Faculty of Medicine; ${ }^{4}$ Pediatrics, Yıldırım Beyazıt University, Faculty of Medicine, Ankara, Turkey

Background and Aim To investigate the effect of surgery time on prognosis of newborns with meningomyelocele.

Methods The records of neonates with meningomyelocele were retrospectively analyzed. Demographic and clinical characteristics as well as information, timing of surgery, and durations of hospital stay and antibiotic therapy were recorded.

Results The records of 30 babies were included in the final analysis. Overall, the mean gestational age was $37.7 \pm 2.7$ weeks, with a mean birth weight of $2967 \pm 755 \mathrm{~g}$ and head circumference of $35.8 \pm 3.8 \mathrm{~cm}$. In terms of localization, $46.6 \%$ of the meningomyeloceles were lumbosacral, $40 \%$ were lumbar, $10 \%$ were thoracolumbar and $3.3 \%$ were thoracal. The mean size of the meningomyelocele sacs was $4.33 \pm 1.2 \mathrm{~cm}$. Newborns underwent surgery on average of $8.2 \pm 5.9$ days after birth, with an overall mean duration of hospital stay of $30 \pm 25.1$ days. Patients were divided into two groups based on timing of surgery (group $1, \leq 5$ days; group 2, $>5$ days), and comparisons between groups revealed that earlier surgery was associated with significantly shorter durations of hospital stay $(p<0.001)$ and antibiotic therapy $(\mathrm{p}<0.05)$. 
Conclusion Early surgical intervention ( $\leq 5$ days) was associated with a shorter duration of hospital stay and antibiotic therapy as well as a lower complication rate. We recommend that corrective surgery be undertaken as soon as reasonably possible.

\section{OCCIPITAL LOBE EPILEPSY IN KUWAITI CHILDREN: A STUDY OVER 2 YEARS}

doi:10.1136/archdischild-2012-302724.1498

MZ Chinnathambi, SK Cheruveettara, A Moosa. Pediatrics, Adan Hospital, Kuwait, Kuwait

Objective To study the clinical profile and therapeutic outcome of occipital lobe epilepsy in Kuwaiti children attending epilepsy clinic in a regional hospital.

Design A retrospective case series study over two years.

Setting Analysis of case files from the epilepsy clinic of a regional hospital who were diagnosed with occipital epilepsy between Aug 2009 to Aug 2011

Subjects Eleven cases were retrieved for study.

Results Ten children had the late childhood form of Gastaut (age 6-12 years) and one had the early childhood form of Panayiotopoulos (age 3 years). Ten children had visual symptoms all of whom presented with sudden onset and cessation of blindness lasting for seconds to few minutes and 5 had visual hallucinations. Half of them had headache. Ten were idiopathic and one of them had focal occipital encephalomalacia. The EEG showed occipital spikes in 8 cases, extraoccipital spikes in 2 cases and generalized discharges in 1 case. All the patients responded well to antiepileptic drugs (carbamazepine, oxcarbazepine, sodium valproate, leviteracetam).

Conclusion Occipital epilepsy, though rare must be considered in children with paroxysms of transient visual symptoms with or without head ache and must be distinguished from migraine.

\section{STUDY OF THE PATTERN OF CEREBRAL PALSY AMONG CHILDREN IN ALEXANDRIA, EGYPT}

doi:10.1136/archdischild-2012-302724.1499

HH Abdeldayem, F Kamel, M Khalil, S Elmansy. Pediatrics, Pediatric Neurology Unit, Faculty of Medicine, Alexandria University, Alexandria, Egypt

Cerebral Palsy (CP) is the commonest neuromotor impairment in childhood. In recent years, there has been mounting changes in $\mathrm{CP}$ pattern. Advances in perinatal and newborn cares have been responsible for these changes. Advances in medical techniques have been also responsible for the extension of the life of CP children to an age of diagnosis and classification. Over the last several years, our knowledge about CP has been substantially improved, but much works still remains. Despite of its importance, the problem is largely ignored in developing countries including Egypt. The aim of our study was arranged for:

1. Giving a recent comprehensive study about CP patterns among CP infants and children attending Alexandria University Children's Hospital over a period of one year (20102011) including: clinical types, topographic types, etiology, and severity.

2. Identifying common associated impairments of CP cases.

3. Comparing our results with other similar studies in Egypt and other developing and developed countries.

\section{OXIDATIVE STRESS IN IRANIAN EPILEPTIC CHILDREN}

doi:10.1136/archdischild-2012-302724.1500

MH Dehghan. Biochemistry, Alborz University of Medical Sceinces, Karaj, Iran

Background Lipid peroxidation is an indicator of free radical metabolism and oxidative stress in human beings and other organisms. Malondialdehyde (MDA), an end product of lipid peroxidation, is a metabolite that can be readily estimated in serum samples. Our objective in this study was to ascertain the variations in alondialdehyde (MDA) in chidren with epilepsy.

Material and Methods This study was carried out in Ardabil and Albroz provinces of Iran after obtaining clearance from the Institutional Ethics Committee. Informed consent was obtained from all the subjects. The quantitative examination of MDA was performed according to standard procedures. The ideal serum level of MDA is below $2.5 \mathrm{nmol} / \mathrm{ml}$.

Results 90 children with confirmed epilepsy (mean age $9.3 \pm 1.5$ ) were included in the study. MDA levels ranged from 1.5 to 3.6 $\mathrm{nmol} / \mathrm{ml}$ (mean level $=2.03 \pm 0.37 \mathrm{nmol} / \mathrm{ml}$ ). Eighty children $(90 \%)$ had MDA levels above the upper limit of normal. Ten patients had levels above $2.85 \mathrm{nmol} / \mathrm{ml}$.

Conclusions This study had shown that the estimation of MDA levels in serum is a convenient method to study lipid peroxidation and thereby oxidative stress in children with epilepsy. Over half of children with epilepsy have excess oxidative stress as indicated by high levels of MDA in the serum. Correlations between MDA level and characteristics of epilepsy, AED therapy, nutritional status and other medical conditions need to be observed in a larger cohort.

\section{QUALITATIVE STUDY OF PSYCHOSOCIAL PROBLEMS AMONG MOTHERS OF CHILDREN WITH CEREBRAL PALSY ATTENDING TERTIARY CARE HOSPITALS IN WESTERN INDIA}

doi:10.1136/archdischild-2012-302724.1501

1.2SM Nimbalkar, ${ }^{3} \mathrm{~S}$ Raithatha, ${ }^{1} \mathrm{RA}$ Shah, ${ }^{2} \mathrm{D}$ Antani. 'Department of Pediatrics, Pramukhswami Medical College; ${ }^{2}$ Central Research Services, Charutar Arogya Mandal, ${ }^{3}$ Department of Community Medicine, Pramukhswami Medical College, Anand, India

Background and Aims The parents of children with cerebral palsy face several problems in meeting physical, psychological and social requirements of their children. We explored the psychosocial problems faced by the mothers of children with cerebral palsy in rural and urban settings and compared the same.

Methods Qualitative research design through Focus Group Discussions (FGDs) was utilized. Two FGDs were conducted - one each at rural and urban tertiary level care hospital involving eight and six mothers respectively. Ethical clearance was obtained from Institutional Ethics Committee. The FGDs were conducted by a moderator using an FGD guide and were video recorded. Transcripts were prepared from the video recordings and were analyzed qualitatively.

Results The problems experienced by the mothers were associated with common themes such as - Disturbed social relationships, Physical problems, Moments of Happiness, Worries about future, Need for a special school, Requirement of a support group, Lack of adequate number of trained physiotherapists and Lack of government support. All the parents had children with problems since birth and most had gone to various health care providers for a cure for their child. The participants in the FGD undertaken at the urban based hospital appeared to be poorly adjusted to the problem of the child as compared to the participants in the rural based hospital

Conclusions Current pyscho-social status of the parents should always be considered. Program planners, Hospital administrators and health care providers need to be sensitized about the perceived need of this vulnerable population.

1502 MALFORMATIONS OF CORTICAL DEVELOPMENT AND EPILEPSY: A REPORT OF 13 CASES

doi:10.1136/archdischild-2012-302724.1502 